\title{
A prospective randomized study on limits of colposcopy and histology: the skill of colposcopist and colposcopy-guided biopsy in diagnosis of cervical intraepithelial lesions
}

\author{
Giuseppe Bifulco ${ }^{1 *+}$, Nicoletta De Rosa ${ }^{1+}$, Giada Lavitola ${ }^{1}$, Roberto Piccoli ${ }^{1}$, Alessandra Bertrando ${ }^{1}$, \\ Valentina Natella ${ }^{2}$, Costantino Di Carlo ${ }^{1}$, Luigi Insabato ${ }^{2}$ and Carmine Nappi ${ }^{3}$
}

\begin{abstract}
Background: The main objective of our study was to evaluate the colposcopist ability to correctly identify the worst area of a cervical lesion where biopsy should be performed; the secondary objective was to investigate the influence of the colposcopist skill in grading cervical preneoplastic lesions.

Methods: 296 patients referred for colposcopy were enrolled in a prospective study. All patients were randomized in two groups: in the first group, "senior group", the colposcopy was performed by an experienced colposcopist; in the second group, "junior group", the colposcopy was performed by a less experienced colposcopist. A detailed colposcopic description, including a grading of the lesion, was completed for each case. During the colposcopic exam patients underwent two direct biopsies; each biopsy was labeled with letter A (suspicious area with most severe grade) or B (suspicious area with less severe grade) according to the judgment of the colposcopist. An experienced pathologist reanalyzed the histological slides, after routine diagnosis.

Results: The senior group identify the worst area of the cervical lesion in statistical significant higher rates than junior group. Specimen A resulted representative of the higher-grade lesion (A>B) in $73.7 \%(N=28)$ in senior group and in $48.4 \%(N=15)$ in junior group; while in $26.3 \%(N=10)$ the higher-grade lesion corresponded to specimen $B(A<B)$ in senior group and in $51.6 \%(N=16)$ in junior group $(p<.05)$.
\end{abstract}

Conclusion: The ability of a colposcopist in grading cervical lesion depends on his experience.

Keywords: Cervical intraepithelial lesions, Multiple biopsies, Colposcopic accuracy, Colposcopic grade

\section{Background}

Defining the presence, the extension and the severity of cervical intraepithelial neoplasia (CIN) is an important clinical issue in reducing cervical cancer risk and development. Colposcopy represents the second step of the diagnostic approach $[1,2]$. One of the main roles of

\footnotetext{
* Correspondence: giuseppe.bifulco@unina.it

${ }^{\dagger}$ Equal contributors

'Department of Neuroscienze e Scienze Riproduttive ed

Odontostomatologiche, University of Naples "Federico II", Naples, Italy

Full list of author information is available at the end of the article
}

colposcopy is to guide the diagnostic biopsy. The result of the histological exam performed on the cervical biopsy is then considered as the best diagnosis in the preoperative approach to CIN.

Colposcopic accuracy varies according to the skill of the colposcopist, the age of the patients and the grade of the lesions. Mitchell and colleagues in a meta-analysis, reported that the sensitivity of colposcopy ranged from 64 to $99 \%$ and the specificity from 30 to $93 \%$ [3]. Indeed, colposcopic assessment more often overestimated the severity of the lesions.

\section{Biomed Central}

(c) 2015 Bifulco et al. Open Access This article is distributed under the terms of the Creative Commons Attribution 4.0 International License (http://creativecommons.org/licenses/by/4.0/), which permits unrestricted use, distribution, and reproduction in any medium, provided you give appropriate credit to the original author(s) and the source, provide a link to the Creative Commons license, and indicate if changes were made. The Creative Commons Public Domain Dedication waiver (http://creativecommons.org/publicdomain/zero/1.0/) applies to the data made available in this article, unless otherwise stated. 
Mistakes in cervical histological findings on bioptic specimens, have also been documented, $[4,5]$ and histological CIN diagnoses are not entirely reproducible $[6,7]$. Indeed, similarly to cytological interpretation, histological assessment of cervical dysplasia is complicated by interobserver variability [8]. The strongest source of disagreement was the threshold between normal and CIN 1. Agreement was higher for CIN 3 than for CIN 2 [9]. Moreover, the proportion of false-positive diagnoses of CIN 2 or worse varied according to cytologic and HPV test results [10].

Many studies investigated the correlation between histological diagnosis from colposcopically directed punch biopsies and definitive diagnosis after conization or hysterectomy $[11,12]$. Only few authors concluded that directed cervical biopsies provide a consistent estimate of the final grading of CIN lesions [13, 14], whereas most of the studies showed just a moderate correlation. A complete agreement between biopsy and cone specimen was reported in no more than 43- $51 \%$ of cases [11, 12, 15-19]. About 14-24 \% LEEP specimens were negative for dysplasia. Giannella L. et al. showed that a severe cervical lesion (CIN2) with a minor colposcopic impression may predict a lower grade lesion on cone specimen [20].

One of the potential explanations for negative LEEP findings following a biopsy diagnosis of HSIL includes misdiagnosis of the original biopsy [21].

The choice of cervical point where to perform the biopsy is crucial to obtain a proper diagnosis. Conventionally biopsy must be performed in the worst area of cervical lesion, and should be representative of the entire lesion.

The main aim of this study was to investigate the influence of the skill of the colposcopist in correctly grading cervical preneoplastic lesion. Moreover, we investigated how good are skilled and junior colposcopists in identifying the worst area of a cervical lesion where biopsy should be performed.

\section{Methods}

A prospective randomized study was carried out from January 2012 to October 2014, in the Unit of CervicoVaginal Pathology of the Department of Obstetrics and Gynecology of the University Hospital Federico II in Naples, Italy.

All women referred for colposcopic examination and undergoing cervical biopsy under colposcopic guidance were invited to participate in this study. Our Institutional Review Board approved the protocol of the study and the study was conducted according to the guidelines of the Declaration of Helsinki (1975).

After signing their informed consent, all patients with a positive cytology, were randomly assigned to two main groups (junior colposcopists and senior colposcopists) corresponding to three junior colposcopists (i.e. a postgraduate physician with one-year experience in a Unit of Cervicovaginal Pathology) and three senior colposcopists (i.e. a trained gynecologist with at least 5 years of practice in a second level Unit of Colposcopy and Cervicovaginal Pathology). A physician who was not involved in the examination used the computer-generated list to assign each patient to a colposcopist.

Colposcopy and guided cervical biopsies were performed in a single procedure.

Patients were eligible for enrolment according to both the following criteria:

- satisfactory colposcopy (squamo-columnar junction fully visible) with atypical transformation zone (aceto-white epithelium).

- aceto-white lesion extending for 2 or more quadrants (allowing the execution of a double biopsy).

A colposcopic suspect for invasive cervical cancer and pregnancy (which can alter colposcopic findings), were considered as exclusion criteria.

During colposcopic examinations, after application of $3 \%$ acetic acid, all visible lesions were classified according to the 2011 Colposcopic Terminology of the International Federation for Cervical Pathology and Colposcopy [22].

The examiner performed, for each patient, two guided biopsies using cervical biopsy forceps with 5 - to $6-\mathrm{mm}$ jaws, yielding 3- to 4-mm biopsies. The two specimens were placed into two different vials of fixative.

An extensive description of the two cervical sites, where biopsies were performed, was recorded; in particular, the examiner specified:

1. the site of biopsies (dividing the cervix into 4 quadrants by 2 perpendicular lines drawn from 12 to 6 o'clock, and from 9 to 3 o'clock);

2. the grading (grade 1-minor or 2-major) and the colposcopic features (thin/dense aceto-white epithelium with fine/coarse punctuation or mosaic);

3. which biopsy was considered the most suspicious and representative of the whole cervical lesion (biopsy A) and which biopsy, performed on a less severe area of the lesion, was considered additional but not required to obtain histological diagnosis (biopsy B).

All cervical biopsies were firstly examined by the pathologist on duty at the Pathology Laboratory, who was unaware of the study. The specimens were composed of small or tiny fragments of cervical tissue. Two serial 4$\mu \mathrm{m}$ sections of formalin-fixed, paraffin embedded 
samples were stained with hematoxylin and eosin. The specimens were classified according to the World Health Organization criteria as normal, CIN 1, CIN 2, CIN 3/ carcinoma in situ or micro-invasive carcinoma [23].

At the end of the study all the histologic sections were reviewed by an experienced gynecologic pathologist (LI). In uncertain cases immunohistochemical stains were performed with labeling index for Ki67 to evaluate the proliferative activity, and for 16 protein expression to determine the different degrees of CIN. 4- $\mu \mathrm{m}$ serial sections from representative blocks were cut, mounted on poly-L-lysine coated glass slides and used for the immunohistochemical staining for ki67 and p16 protein. Representative sections were incubated with the primary antibodies, overnight at $4{ }^{\circ} \mathrm{C}$. Subsequently, the slides were incubated with biotinylated secondary antibodies, peroxidase-labelled streptavidin (DAKO LSAB kit HRP, Carpinteria, CA) and chromogenic substrate diaminobenzidine (DAB, Vector Laboratories, Burlingame, U.S.A.) for the development of the peroxidase activity. Slides were counterstained with hematoxylin, dehydrated and cover-slipped with a synthetic mounting medium (Entellan, Merck, Germany).

The experienced pathologist was blinded to the referral cytology and the colposcopic examination. The histology of the most severe lesion (specimen A or B) was recoded as the final diagnosis. Although some patients underwent cervical conization or loop electrosurgical excision procedure (LEEP) as treatment for cervical neoplasia, results of these procedures were not considered in determining the final diagnosis.

\section{Statistical analysis}

Statistical analysis was performed using SPSS software (version 20; SPSS, Inc. Chicago, IL, USA).

To compare demographic and clinical data between the two groups (senior group and junior group) Student's t-test and Mann-Whitney test were used.

The main endpoint was to test the hypothesis that expert colposcopists may perform with a higher degree of accuracy the guided cervical biopsy. If this were true, expert colposcopists would identify the worse biopsy in a higher percentage of cases in comparison to junior colposcopists.

Differences in proportions were tested with $x^{2}$ test (sites of biopsies, histological diagnosis rates, gradation of colposcopist judgment attributed to biopsy sites) and with Wilcoxon's signed rank test (routine versus revision histological analysis). Statistical analysis for colposcopist evaluation attributed to biopsy sites was performed considering only patients with definitive diagnosis of CIN. A colposcopist evaluation reporting $A=B$ was considered not informative and excluded from the statistical analysis. The level of significance for these tests was set at $p<0.05$.
The significance of the association between colposcopic grading and biopsy histology was determined using $X^{2}$ test, while the strength of the association was assessed using $\mathrm{k}$ statistics. Prior to calculating the $\mathrm{K}$ values the histological diagnosis were dichotomized into two classifications: Negative/Cervicites/Metaplasia/koilocytosis /Condylomatosis/CIN 1 and CIN 2/CIN 3. Standard definitions were used to interpret the $\kappa$ statistics [24].

\section{Results}

A total of 296 gave their consent to participate in this study. At the time of colposcopy, 41 patients were excluded as they did not satisfy the inclusion criteria: 18 women had unsatisfactory or negative colposcopy, 19 women had limited extension of lesion involving $0-1$ quadrant of the cervix and 4 women had a suspected invasive cervical cancer. Among the 255 enrolled patients in 4 cases one or both biopsy specimens were insufficient for a diagnosis. Therefore, 251 cases met all criteria for analysis according to the study protocol (Fig. 1).

The mean age of patients was 32.4 years (range 19-52; $\mathrm{SD} \pm 8.5)$.

One hundred twenty seven cases were randomized into senior group (50.6\%), while 124 cases (49.4\%) into junior group. Demographic characteristics of the patients, indications for colposcopy, colposcopic grade and final histological diagnosis are shown in Table 1 . No significant differences for age, parity, educational level, colposcopic indications, colposcopic grade and final histological diagnosis were found between the two groups (Table 1).

Data regarding histological diagnosis performed by the routine practice pathologist and by the experienced gynaecologic pathologist for specimen $\mathrm{A}$ and $\mathrm{B}$ are shown in Table 2. A significant statistical difference was found between routine and revised histological analysis only for specimen B ( $p=.03)$ (Table 2).

Considering only patients with definitive histological diagnosis of CIN (diagnosis performed by the expert pathologist) the senior group identify the worst area of the cervical lesion in statistical significant higher rates than junior group. Specimen A resulted representative of the higher-grade lesion $(\mathrm{A}>\mathrm{B})$ in $73.7 \%(N=28)$ in senior group and in $48.4 \%(N=15)$ in junior group; while in $26.3 \%(N=10)$ the higher-grade lesion corresponded to specimen $\mathrm{B}(\mathrm{A}<\mathrm{B})$ in senior group and in $51.6 \%(N=16)$ in junior group (Table $3, p<.05)$. The difference was significant both in routine than in revised histological analysis (Table 3, $p<.05$ ).

A significant difference was also found in rate of colposcopist evaluations between groups when stratified by colposcopic findings of grade 1 and 2 (Table 4). Indeed, in presence of grade 1 lesions, junior colposcopist identified in A a less severe lesion than in B in a significant higher rate of cases than in senior group (A<B: $70.0 \%$ 


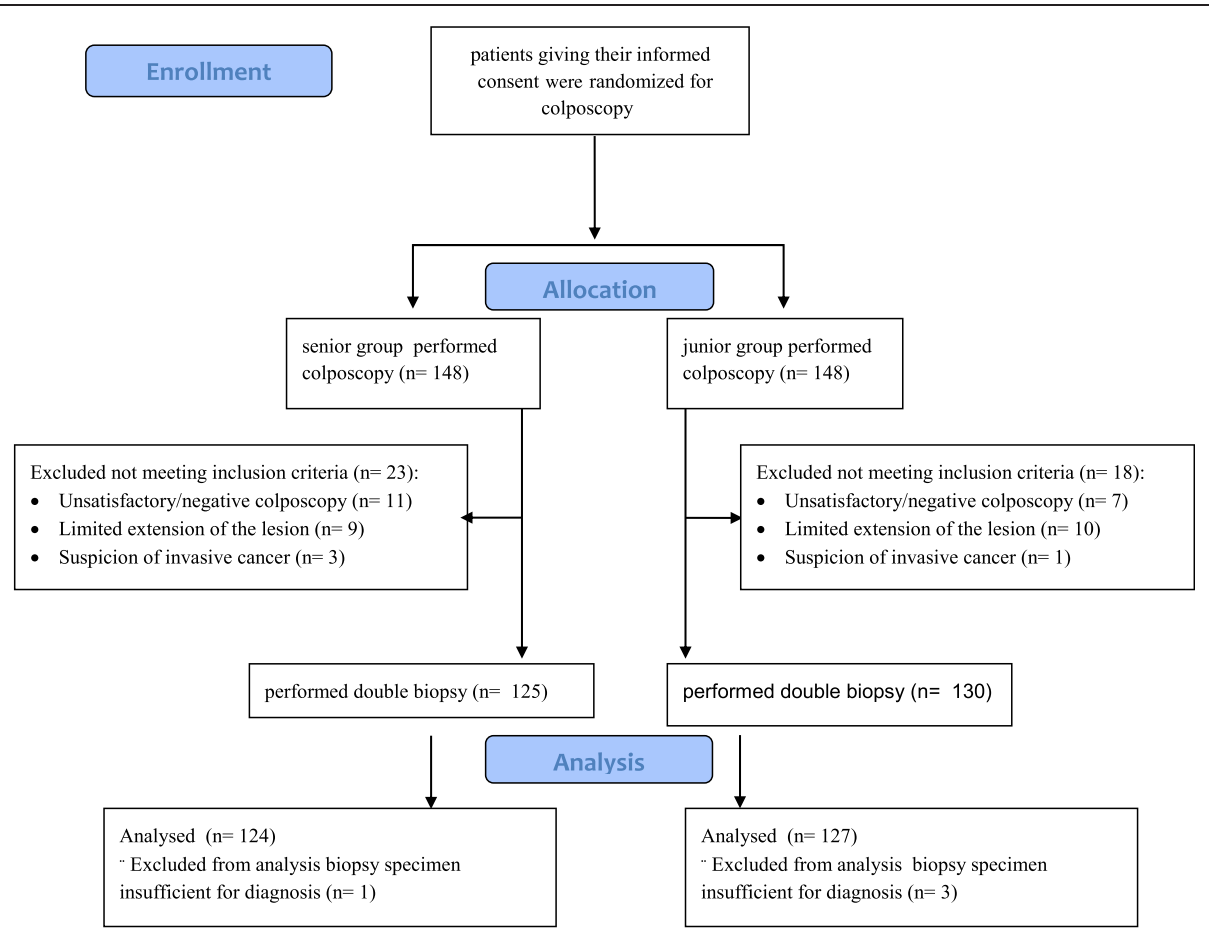

Fig. 1 Patients enrolment and randomization

Table 1 Patient demographic characteristics, colposcopic indication and findings, histological diagnosis for two groups

\begin{tabular}{|c|c|c|c|c|}
\hline & & junior group $(N=124)$ & senior group $(N=127)$ & $p$ Value \\
\hline Age (years \pm S.D.) & & $32.2 \pm 8.1$ & $32.6 \pm 8.9$ & .23 \\
\hline Parity ( \pm S.D.) & & $0.62 \pm 0.92$ & $0.57 \pm 0.85$ & .69 \\
\hline \multirow[t]{5}{*}{ Educational level } & & & & .82 \\
\hline & Elementary education & $3(2.4)$ & $2(1.6)$ & \\
\hline & Lower secondary education & $50(40.3)$ & $51(40.2)$ & \\
\hline & Upper secondary education & $58(46.8)$ & $60(47.2)$ & \\
\hline & Postsecondary education & $13(10.5)$ & $14(11.0)$ & \\
\hline
\end{tabular}

Colposcopic Indication ${ }^{\mathrm{b}}$

$\begin{array}{lll}\text { ASC-US/ASC-H } & 35(27.6) & 32(25.8) \\ \text { AGC-NOS } & 4(3.1) & 5(4.0) \\ \text { L-SIL } & 57(44.9) & 66(53.2) \\ \text { H-SIL } & 31(24.4) & 21(16.9)\end{array}$

Colposcopic Grade

$\begin{array}{lll}\text { TAG1 } & 104(83.9) & 96(75.6) \\ \text { TAG2 } & 20(16.1) & 31(24.4)\end{array}$

$\begin{array}{ll}56(45.2) & 61(48) \\ 39(31.5) & 33(26) \\ 29(23.4) & 33(26)\end{array}$

CIN1 / Koilocytosis / Condylomatosis

$29(23.4)$

${ }^{\mathrm{a}}$ In senior group colposcopic examination and biopsies were performed by experienced colposcopists; in junior group post-graduate doctors with one-year experience in Unit of Cervicovaginal Pathology performed the diagnostic procedures

bIndications for colposcopy: Atypical squamous cells of undetermined significance (ASC-US); Atypical squamous cells - cannot exclude HSIL (ASC-H); Atypical Glandular Cells not otherwise specified (AGC-NOS); Low grade squamous intraepithelial lesion (LSIL); High grade squamous intraepithelial lesion (HSIL)

'TAG1: Atypical Transformation of Grade 1, TAG2: Atypical Transformation Grade 2 
Table 2 Histological diagnosis of specimen A and B resulted from the routine analysis and from the revision analysis performed by an experienced gynecologic pathologist

\begin{tabular}{|c|c|c|c|c|c|c|}
\hline Hystological Diagnosis & Specimen A N $(\%$ & & & Specimen B N (\% & & \\
\hline & Routine Analysis & Revision Analysis $^{a}$ & $p$ Value & Routine Analysis & Revision Analysis $^{a}$ & $p$ Value \\
\hline Negative & $18(7.2)$ & $40(15.9)$ & .34 & $35(13.9)$ & $51(20.3)$ & .03 \\
\hline Cervicites / Metaplasia & $116(46.2)$ & $96(38.2)$ & & $95(37.8)$ & $102(40.6)$ & \\
\hline CIN1 / Koilocytosis / Condylomatosis & $71(28.3)$ & $65(25.9)$ & & $73(29.1)$ & $52(20.7)$ & \\
\hline CIN2 & $20(8.0)$ & $14(5.6)$ & & $24(9.6)$ & $10(4.0)$ & \\
\hline CIN3 & $26(10.4)$ & $36(14.3)$ & & $24(9.6)$ & $36(14.3)$ & \\
\hline
\end{tabular}

${ }^{a}$ Revision analysis: analysis performed by experienced pathologist. In uncertain cases immunohistochemical stains were used, particularly, antibody against ki67 to evaluate the proliferative activity and p16 protein expression to determine the different degrees of CIN

vs. $36 \%, p=.01)$. The difference was not significant in grade 2 lesions (Table 4 ).

The association between histological diagnosis and colposcopic grade was highly significant $(\mathrm{p}<.001)$ and the strength of the correlation, as assessed by the $\mathrm{K}$ statistics, was fair for each specimen (A or B) and histological analysis ( $\mathrm{k}=0.32$; CI $95 \%$ : .16-.47; and $\mathrm{\kappa}=0.30$; CI $95 \%$ : $.17-.44$, for routine and revised analysis respectively) (Table 5). The highest $\mathrm{K}$ value was observed in senior group ( $\kappa=.42$; CI, $95 \%$ : .25-.62) (Table 5). In junior group, the association between histological diagnosis and colposcopic grade was shown significant $(p<0.05)$ but the strength of this association was found slight $(\kappa=.20$; CI $95 \%:-.01-.40$ ) (Table 5).

\section{Discussion and conclusion}

This study prospectively investigates the ability of colposcopist in grading and performing diagnosis of a CIN lesion in uterine cervix.

Our data show a high significant correlation between colposcopic grading and histologic grading in single and in double biopsy both in routinely and in revision analysis as well.

This high correlation was lost when colposcopy was performed by less experienced examiner (junior Group). On the other hand, in senior group exact agreement was found in $85.1 \%$ of grade 1 lesions and in $51.5 \%$ of grade 2 lesions. Accordingly, the strength of this correlation, as assessed by $\kappa$ statistics, is fair $(\kappa=.42)$ in senior Group and slight in junior group $(\kappa=.20)$. Baum ME el al. [25] and Benedet JL. et al. [26] have shown similar data in $\mathrm{k}$ statistic according to examiners experience. Overall the highest difficulty both in senior than junior group was the identification of grade 2 lesions.

In Benedet's report, the association between Pap smear cytology and colposcopic impression has been found higher significant than association between punch biopsy histology and colposcopy, indeed the strength of this correlation was moderate $(\kappa=.56)$ [26]. However, also the correlation between cervical punch biopsy and LEEP biopsy was moderate $(\kappa=.44)$ or even fair $(\kappa=.31)$ [25-28].

The value of multiple or random cervical biopsies at the time of colposcopy for evaluation of an abnormal cytology has been discussed in the last decades. The number of specimens seems to influence the sensitivity of the diagnosis on cervical biopsies. The proportion of women with CIN 2 or worse increased when multiple random cervical biopsies in quadrants without lesions were performed [27]. Zuchna C. et al. [29] showed that two biopsies achieved a highly significant improvement in agreement between punch biopsy and cone specimen in comparison to one biopsy. On the contrary, in our

Table 3 Gradation of colposcopist judgment attributed to biopsy sites corresponding to specimen A and B before and after revision analysis in senior and junior group

\begin{tabular}{|c|c|c|c|c|}
\hline Examiner group $^{a}$ & Colposcopist evaluation $^{\text {b }}$ & senior N (\%) & junior N (\%) & $p$ value \\
\hline \multirow[t]{2}{*}{ Routine histological Analysis } & $A<B$ & $10(26.3)$ & $16(51.6)$ & .03 \\
\hline & $A>B$ & $28(73.7)$ & $15(48.4)$ & \\
\hline \multirow[t]{2}{*}{ Revised histological Analysis ${ }^{c}$} & $A<B$ & $12(27.3)$ & $19(50.0)$ & .03 \\
\hline & $A>B$ & $32(72.7)$ & $19(50.0)$ & \\
\hline
\end{tabular}

Statistical analysis was performed considering only patients with definitive diagnosis of CIN. A colposcopist evaluation reporting $A=B$ was considered not informative and excluded from the statistical analysis

a In senior group colposcopic examination and biopsies were performed by experienced colposcopists; in junior group post-graduate doctors with one-year experience in Unit of Cervicovaginal Pathology performed the diagnostic procedures

${ }^{b}$ According to the judgment of the colposcopist biopsy A was considered the most suspicious and representative of the whole cervical lesion and biopsy B was considered additional but not required to obtain histological diagnosis

${ }^{c}$ Revision analysis: analysis performed by experienced pathologist. In uncertain cases immunohistochemical stains were used, particularly, antibody against ki67 to evaluate the proliferative activity and p16 protein expression to determine the different degrees of CIN 
Table 4 Gradation of colposcopist judgment attributed to biopsy sites corresponding to specimen A and B stratified for colposcopic grading of the lesion in senior and junior group

\begin{tabular}{|c|c|c|c|c|}
\hline \multirow{2}{*}{$\begin{array}{l}\text { Colposcopic } \\
\text { Grading }\end{array}$} & \multirow{2}{*}{$\begin{array}{l}\text { Colposcopist } \\
\text { evluation }^{b}\end{array}$} & \multicolumn{2}{|c|}{ Examiner Group ${ }^{a}$} & \multirow[t]{2}{*}{$p$ value } \\
\hline & & senior group & junior group & \\
\hline \multirow[t]{2}{*}{ TAG1 } & $A<B$ & $9(36.0)$ & $21(70.0)$ & \multirow[t]{2}{*}{.01} \\
\hline & $A>B$ & $16(64.0)$ & $9(30.0)$ & \\
\hline \multirow[t]{2}{*}{ TAG2 } & $A<B$ & $5(20.0)$ & $0(0.0)$ & \multirow[t]{2}{*}{.09} \\
\hline & $A>B$ & $20(80.0)$ & $12(100.0)$ & \\
\hline
\end{tabular}

Statistical analysis was performed considering only patients with definitive diagnosis of $\mathrm{CIN}$. A colposcopist evaluation reporting $\mathrm{A}=\mathrm{B}$ was considered not informative and excluded from the statistical analysis.

${ }^{\text {a } I n ~ s e n i o r ~ g r o u p ~ c o l p o s c o p i c ~ e x a m i n a t i o n ~ a n d ~ b i o p s i e s ~ w e r e ~ p e r f o r m e d ~ b y ~}$ experienced colposcopists; in junior group post-graduate doctors with one-year experience in Unit of Cervicovaginal Pathology performed the diagnostic procedures.

${ }^{\mathrm{b}}$ According to the judgment of the colposcopist biopsy A was considered the most suspicious and representative of the whole cervical lesion and biopsy $B$ was considered additional but not required to obtain histological diagnosis. 'TAG1: Atypical Transformation of Grade 1, TAG2: Atypical Transformation Grade 2.

study, second biopsy did not increase the strength of correlation between colposcopic and histological grade neither in senior nor in junior group.

Colposcopic findings judged more representative of CIN lesion are presence of mosaics and punctuation both in colposcopic grade 1 and 2, while thin or dense aceto-white epitelium without vascular pattern are considered by colposcopist less representative of lesion grade. The ability of colposcopist in differentiating and grading two point of the same cervical lesion is limited.
In presence of a CIN lesion, the rate of colposcopic evaluations in biopsy sites (A and B) correct $(\mathrm{A}>\mathrm{B})$, was $62 \%$, on the contrary in $38 \%$ of cases colposcopists do not identify the worst area of the cervical lesion $(\mathrm{A}<\mathrm{B})$.

Massad et al. [30] previously reported that colposcopic impression, colposcopic features as color, margin, vascularity and modification of Reid index do not discriminate between acetowhite lesion that arbor CIN2+ and those that not. However, the failure to detect CIN2+ lesion at colposcopy may reflects a measurable physical characteristic of the dysplastic epithelium. Yang B. et al. demonstrate that false negative colposcopic impression is sometimes secondary to the relative thinness of some CIN2-3 lesion [31].

The colposcopist experience influences significantly colposcopic accuracy in grading cervical lesion and in identify its worst area; indeed, junior colposcopist showed more difficulties in identify the worst area of the lesion, in about half of cases they fail in the classification of the lesion.

Colposcopic grade influence colposcopist judgment in less expert colposcopists; higher colposcopic lesion grade (grade 2) is associated with higher rate of correct evaluation in junior group $(\mathrm{A}>\mathrm{B})$. On the contrary junior colposcopist fail, significantly more than senior colposcopist, in identification of worst area was to perform biopsy in grade 1 lesion. Probably this is due to the larger extension of high-grade lesion and to the coexistence of low-grade area in high-grade lesion.

Overall, $42.2 \%$ of histological reports have been modified after revision by experienced gynecologic pathologist:

Table 5 Association and strength of correlation between histological diagnosis and colposcopic grading in senior and junior group

\begin{tabular}{|c|c|c|c|c|}
\hline & & Histological Diagnosis ${ }^{\mathrm{b}}$ & & $p$ value ${ }^{c} \mathrm{~K}$ Value; $95 \%$ C.I. \\
\hline Specimen & Colposcopic Grading $^{a}$ & Negative/CIN1 N (\%) & CIN2/3 N (\%) & \\
\hline \multicolumn{5}{|c|}{ Routine analysis after single biopsy (A) } \\
\hline \multirow[t]{2}{*}{ total group } & TAG1 & $176(85.9)$ & $24(52.2)$ & $<.0010 .32 ; .16-.47$ \\
\hline & TAG2 & $29(14.1)$ & $22(47.8)$ & \\
\hline \multirow[t]{2}{*}{ senior group } & TAG1 & $87(84.5)$ & $9(37.5)$ & $<.0010 .42 ; .25-.62$ \\
\hline & TAG2 & $16(15.5)$ & $15(62.5)$ & \\
\hline \multirow[t]{2}{*}{ junior group } & TAG1 & $89(87.3)$ & $15(68.2)$ & $<.050 .20 ;-.01-.40$ \\
\hline & TAG2 & $13(12.7)$ & $7(31.8)$ & \\
\hline \multicolumn{5}{|c|}{ Revision analysis after two biopsies (A and B) } \\
\hline \multirow[t]{2}{*}{ total group } & TAG1 & $164(86.8)$ & $36(58.1)$ & $<.0010 .30 ; .17-.44$ \\
\hline & TAG2 & $25(13.2)$ & $26(41.9)$ & \\
\hline \multirow[t]{2}{*}{ senior group } & TAG1 & $80(85.1)$ & $16(48.5)$ & $<.0010 .37 ; .15-.54$ \\
\hline & TAG2 & $14(14.9)$ & $17(51.5)$ & \\
\hline \multirow[t]{2}{*}{ junior group } & TAG1 & $84(88.4)$ & $20(69.0)$ & $<.050 .22 ; .03-.42$ \\
\hline & TAG2 & $11(11.6)$ & $9(31.0)$ & \\
\hline
\end{tabular}

${ }^{\mathrm{a}}$ The histology of the most severe lesion obtained with specimen A or B was recoded as the final histological diagnosis

${ }^{\text {b} T A G 1: ~ A t y p i c a l ~ T r a n s f o r m a t i o n ~ o f ~ G r a d e ~ 1, ~ T A G 2: ~ A t y p i c a l ~ T r a n s f o r m a t i o n ~ G r a d e ~} 2$

${ }^{\mathrm{C}}$ The significance of the association between colposcopic grading and histological diagnosis was determined within group using $x^{2}$ test, the strength of the association was assessed using $\mathrm{k}$ statistics. To perform this analysis the histological diagnosis were dichotomized into two classifications: Negative/Cervicites/ Metaplasia/koilocytosis/Condylomatosis/CIN 1 and CIN 2/CIN 3 
$20.8 \%$ cases resulted in a reduction and $33 \%$ showed an increase of lesion's grade, while in $46.2 \%$ the adjustment did not influenced the grade of the lesion. This high rate of changed reports could be attributed to the use of immunochemistry for Ki-67 and p16INK4a antibodies that have been demonstrated in many studies valuable adjunctive aids in diagnosis of difficult cervical biopsy [32-34].

A significant statistical difference was found between routine analysis and revision analysis in specimen $\mathrm{B}$. These data show that the second biopsy, performed in cervical area judged by colposcopist less representative of lesion grade and characterized at colposcopy by the absence of vascular pattern and by less marked signs of lesion makes more difficult in performing histological analysis.

In conclusion, our data suggest that a long time experience in a Colposcopic Unit is fundamental to ensure high accuracy of colposcopy, and that a one year colposcopy training program is not enough to achieve these skills. Also expert colposcopist when performs a guided biopsy may not identify the worst area of a CIN lesion, so neither colposcopic impression nor histology can be used alone to guide management.

The lack of a definitive diagnosis on excisional sample (LEEP or cold knife conizzation) cannot ensure the conclusion that perform a second biopsy have a minimal impact on colposcopy accuracy, further study are necessary to achieve this goal.

\section{Competing interests}

The authors do not have any conflict of interest.

\section{Authors' contribution}

BG and DRN have equally contributed to the elaboration of the manuscript. All the Authors' have given their contributions for the study: particularly, BG and DRN have contributed to conception and design of the study protocol and in the analysis and interpretation of the data, NC has supervised the proper conduct of the study and he has revised the article critically. BG, DCC, and PR are the senior colposcopists; BA, DRN, and LG are the junior colposcopists. IL is the experienced gynecologic pathologist and has contributed to draft and revise the study. NV gave substantial contribution in the acquisition of data. All authors read and approved the final manuscript.

\section{Author details}

'Department of Neuroscienze e Scienze Riproduttive ed Odontostomatologiche, University of Naples "Federico II", Naples, Italy. ${ }^{2}$ Department of Advanced Biomedical Science, University of Naples "Federico II", Naples, Italy. ${ }^{3}$ Department of Sanità pubblica, University of Naples "Federico II", Naples, Italy.

Received: 11 June 2015 Accepted: 3 November 2015 Published online: 19 November 2015

\section{References}

1. Wright Jr TC, Massad LS, Dunton CJ, Spitzer M, Wilkinson EJ, Solomon D. 2006 consensus guidelines for the management of women with abnormal cervical screening tests. 2006 ASCCP-Sponsored Consensus Conference. J Low Genit Tract Dis. 2007;11(4):201-22.

2. Partridge EE, Abu-Rustum NR, Campos SM, Fahey PJ, Farmer M, Garcia RL, et al. Cervical cancer screening. National comprehensive cancer networks. J Natl Compr Canc Netw. 2010;8(12):1358-86.
3. Mitchell MF, Schottenfeld D, Tortolero-Luna G, Cantor SB, Richards-Kortum R. Colposcopy for the diagnosis of squamous intraepithelial lesions: a metaanalysis. Obstet Gynecol. 1998;91:626-31.

4. Joste NE, Crum CP, Cibas ES. Cytologic/histologic correlation for quality control in cervicovaginal cytology; experience with 1.582 paired cases. Am J Clin Pathol. 1995;103:32-4.

5. Tritz DM, Weeks JA, Spires SE, Sattich M, Banks H, Cibull ML, et al. Etiologies for non-correlating cervical cytologies and biopsies. Am J Clin Pathol. 1995;103:594-7.

6. Malpica A, Matisic JP, Niekirk DV, Crum CP, Staerkel GA, Yamal JM, et al. Kappa statistics to measure interrater and intrarater agreement for 1790 cervical biopsy specimens among twelve pathologists: qualitative histopathologic analysis and methodology issues. Gynecol Oncol. 2005;99(3 suppl 1):S38-52.

7. Kalof AN, Cooper K. Our approach to squamous intraepithelial lesions of the uterine cervix. J Clin Pathol. 2007;60:449-55.

8. Stoler MH, Schiffman M, The Atypical Squamous Cells of Undetermined Significance-Low-grade Squamous Intraepithelial Lesion Triage Study (ALTS) Group. Interobserver reproducibility of cervical cytologic and histologic interpretations: realistic estimates from the ASCUS-LSIL Triage Study. JAMA. 2001;285:1500-5

9. Dalla Palma P, Giorgi Rossi P, Collina G, NTCC Pathology Group, et al. The reproducibility of CIN diagnoses among different pathologists: data from histology reviews from a multicenter randomized study. Am J Clin Pathol. 2009;132(1):125-32.

10. Dalla Palma P, Giorgi Rossi P, Collina G, NTCC Pathology Group. The risk of false-positive histology according to the reason for colposcopy referral in cervical cancer screening: a blind revision of all histologic lesions found in the NTCC trial. Am J Clin Pathol. 2008;129(1):75-80.

11. Skehan M, Soutter WP, Lim K, Krausz T, Pryse-Davies J. Reliability of colposcopy and directed punch biopsy. Br J Obstet Gynaecol. 1990;97:811-6.

12. Barker B, Garcia F, Lozevski J, Warner J, Hatch K. The correlation between colposcopically directed cervical biopsy and loop electrosurgical excision procedure pathology and the effect of time on that agreement. Gynecol Oncol. 2001;82:22-6.

13. Veridiano NP, Delke I, Tancer ML. Accuracy of colposcopically directed biopsy in patients with cervical neoplasia. Obstet Gynecol. 1981;58:185-7.

14. Cinel A, Oselladore M, Insacco E, Minucci D. The accuracy of colposcopically directed biopsy in the diagnosis of cervical intraepithelial neoplasia. Eur J Gynaecol Oncol. 1990;11:433-7.

15. Howe DT, Vincenti AC. Is large loop excision of the transformation zone (LLETZ) more accurate than colposcopically directed punch biopsy in the diagnosis of cervical intraepithelial neoplasia? Br J Obstet Gynaecol. 1991;98:588-91.

16. Chappatte OA, Byrne DL, Raju KS, Nayagam M, Kenney A. Histological differences between colposcopic directed biopsy and loop excision of the transformation zone (LETZ): a cause for concern. Gynecol Oncol. 1991;43:4650 .

17. Buxton EJ, Luesley DM, Shafi MI, Rollason M. Colposcopically directed punch biopsy: a potentially misleading investigation. Br J Obstet Gynaecol. 1991;98:1273-6.

18. Bonardi R, Cecchini S, Grazzini G, Ciatto S. Loop electrosurgical excision procedure of the transformation zone and colposcopically directed punch biopsy in the diagnosis of cervical lesions. Obstet Gynecol. 1992;80:1020-2.

19. Massad LS, Halperin CJ, Bitterman P. Correlation between colposcopically directed biopsy and cervical loop excision. Gynecol Oncol. 1996;60:400-3.

20. Giannella L, Mfuta K, Gardini G, Rubino T, Fodero C, Prandi S. High-grade CIN on cervical biopsy and predictors of the subsequent cone histology results in women undergoing immediate conization. Eur J Obstet Gynecol Reprod Biol. 2015;186:68-74.

21. Witt BL, Factor RE, Jarboe EA, Layfield LJ. Negative loop electrosurgical cone biopsy finding following a biopsy diagnosis of high-grade squamous intraepithelial lesion: frequency and clinical significance. Arch Pathol Lab Med. 2012;136(10):1259-61.

22. Bornstein J, Bentley J, Bösze P, Girardi F, Haefner H, Menton M, et al. 2011 Colposcopic Terminology of the International Federation for Cervical Pathology and Colposcopy. Obstet Gynecol. 2012;120(1):166-72.

23. Wells M. Epithelial tumors. In: Tavassoli FA, Devilee P, editors. World Health Organization classification of tumors: pathology and genetics of tumors of the breast and female genital organs. Lyons, France: IARC Press; 2003. p. 269-70.

24. Landis J, Koch $\mathrm{G}$. The measurement of observer agreement for categorical data. Biometrics. 1977;33:159-74. 
25. Baum ME, Rader JS, Gibb RK, McAlister RP, Powell MA, Mutch DG, et al. Colposcopic accuracy of obstetrics and gynecology residents. Gynecol Oncol. 2006;103(3):966-70.

26. Benedet JL, Matisic JP, Bertrand MA. An analysis of 84244 patients from the British Columbia cytology-colposcopy program. Gynecol Oncol. 2004:92(1):127-34

27. Pretorius RG, Zhang WH, Belinson JL, Huang MN, Wu LY, Zhang X, Qiao YL. Colposcopically directed biopsy, random cervical biopsy, and endocervical curettage in the diagnosis of cervical intraepithelial neoplasia II or worse. Am J Obstet Gynecol. 2004;191(2):430-4.

28. Moss EL, Hadden P, Douce G, Jones PW, Arbyn M, Redman CW. Is the Colposcopically Directed Punch Biopsy a Reliable Diagnostic Test in Women With Minor Cytological Lesions? J Low Genit Tract Dis. 2012;16(4):421-6.

29. Zuchna C, Hager M, Tringler B, Georgoulopoulos A, Ciresa-Koenig A, Volgger $B$, et al. Diagnostic accuracy of guided cervical biopsies: a prospective multicenter study comparing the histopathology of simultaneous biopsy and cone specimen. Am J Obstet Gynecol. 2010;203(4):321e1-6.

30. Massad LS, Stewarts L, Jeronimo J, Schiffman M. National Institutes of Health/American Society for Colposcopy and Cervical Pathology Research Group. The accuracy of colposcopic grading for detection of high-grade cervical intraepithelial neoplasia. J Low Genit Tract Dis. 2009;13(3):137-44

31. Yang B, Pretorius RG, Belinson JL, Zhang X, Burchette R, Qiao YL. False negative colposcopy is associated with thinner cervical intraepithelial neoplasia 2 and 3. Gynecol Oncol. 2008;110(1):32-6.

32. Klaes R, Benner A, Friedrich T, et al. p16INK4a immunohistochemistry improves interobserver agreement in the diagnosis of cervical intraepithelial neoplasia. Am J Surg Pathol. 2002;26:1389-99.

33. Zhang $Q$, Kuhn L, Denny LA, Ridder R, Herrington $S$, Jenkins $D$, et al. Impact of utilizing p16INK4A immunohistochemistry on estimated performance of three cervical cancer screening tests. Int J Cancer. 2007;120:351-6.

34. Galgano MT, Castle PE, Atkins KA, Brix WK, Nassau SR, Stoler MH. Using biomarkers as objective standards in the diagnosis of cervical biopsies. Am J Surg Pathol. 2010;34(8):1077-87.

\section{Submit your next manuscript to BioMed Central and take full advantage of:}

- Convenient online submission

- Thorough peer review

- No space constraints or color figure charges

- Immediate publication on acceptance

- Inclusion in PubMed, CAS, Scopus and Google Scholar

- Research which is freely available for redistribution 